\title{
Flow-Induced Wave Generated on a Thin Film in a Narrow Gap*
}

\author{
Gaku KUDOU $^{* *}$, Masahiro WATANABE ${ }^{* *}$ and Kensuke HARA ${ }^{* *}$ \\ ** Department of Mechanical Engineering, Aoyama Gakuin University \\ 5-10-1 Fuchinobe, Sagamihara-shi, Kanagawa 229-8558, Japan \\ E-mail: watanabe@me.aoyama.ac.jp
}

\begin{abstract}
This paper deals with a theoretical stability analysis of an unstable wave generated on a thin film subjected to a fluid flow in a narrow gap. In stability analysis, the basic equations of fluid flow around the thin film are based on the Navier-Stokes equations integrated over a gap width between the thin film surface and side wall, assuming that the gap width is enough small compared with a length of the passage. The structural equation of the thin film in the transverse motion is based on the Kirchhoff-Love's thin-plate model. From these basic equations, the governing equations of the thin film coupled with the fluid flow are obtained, employing the moving boundary conditions of the surface of the thin film. These equations are linearized around the equilibrium position, and the dispersion relation of the wave motion is derived as a function of the flow velocity. As a result, the analytical results show that traveling-wave type unstable wave occurs to the thin film due to the fluid flow in the narrow gap and clarify the dispersion relation of the wave motion, phase velocity (traveling-wave velocity) and growth rate of the wave. Moreover, the analytical results are verified by experiments on the most unstable wave number and critical flow velocity.
\end{abstract}

Key words: Vibration Coupled with Fluid Motion, Flutter, Stability Analysis, Self-Excited Vibration, Wave, Flow Induced Vibration

\section{Introduction}

In manufacturing process of flexible materials, such as a paper, sheet and plastic films, the flexible materials are subjected to a fluid flow in a narrow gap for non-contact support and drying. However the interaction of the thin film with the fluid flow causes an unstable wave. The unstable wave reduces the process efficiency and generates noise, in some case, causes fatigue failure to the thin film. For the productivity growth, we need to clarify the instability mechanism and dynamic characteristics of the unstable wave.

Up to this time, many studies on the stability of leakage-flow-induced vibrations and sheet flutter have been reported ${ }^{(1)-(6)}$. Inada and Hayama ${ }^{(1)-(3)}$ studied leakage-flow-induced vibrations of translational one-degree-of-freedom system, and translational-rotational coupled two-degree-of-freedom system, and clarified the instability mechanism of these systems theoretically. It was found that self-excited vibrations occur due to negative fluid damping, when the passage is narrow and divergent shape to the downstream or it includes a constriction at the inlet or a diffuser at the outlet. Nagakura and Kaneko ${ }^{(4)}$ examined the stability of a cantilever beam subjected to the leakage fluid flow by employing the modal analysis method, and they verified the theoretical results comparing with experimental results. Yamaguchi and et al. ${ }^{(5)(6)}$ studied the dynamic behavior and stability of a flexible 
thin sheet subjected to a high-speed fluid flow and they conducted wind tunnel experiments to obtain the critical flow velocity, flutter mode and flutter frequency.

Some studies on the flow induced vibration and flow induced wave of a flexible sheet have been reported ${ }^{(7)-(16)}$. Chang and Moretti ${ }^{(17)}$ studied flow induced vibration of free edges of thin films by using a traveling-wave analysis based on the incompressible potential-flow. In their study, previous studies of edge flutter on two-dimensional waves expands to a more realistic three-dimensional problem, and the simple closed form solutions of the critical flow velocity are presented. Watanabe and Kobayashi ${ }^{(18)(19)}$ studied the stability and dynamic characteristics of a moving flexible sheet with shear fluid flow. In their study, unstable wave occurs to the flexible sheets as a type of traveling-wave due to the shear fluid flow. However, the instability mechanism and dynamic characteristics of an unstable wave of a thin film subjected to a fluid flow in a narrow gap are not yet clarified sufficiently.

This paper presents the theoretical stability analysis of the unstable wave generated on the thin film subjected to the fluid flow in the narrow gap. In the stability analysis, tensile force is applied to the thin film, and the thin film is subjected to air flow in a narrow gap. The analytical results show the stability and wave characteristics of the unstable wave, phase velocity and growth rate of the wave.

\section{Nomenclature}

$\begin{array}{lll}C_{s} & : \text { External damping coefficient } \\ D & : \text { Flexural rigidity }\left(=E h^{3} / 12\right) \\ E & : \text { Young's Modulus } \\ E^{*} & : \text { Internal damping coefficient } \\ f & : \text { Fluid force } \\ f_{s}, f_{w} & : \text { Wall friction coefficients on the film and side wall surface } \\ h & : \text { Film thickness } \\ i & : \text { Imaginary unit }(=\sqrt{-1}) \\ k & : \text { Wave number } \\ L & : \text { Film supporting width } \\ P & : \text { Fluid Pressure } \\ p, P_{0} & : \text { Unsteady and steady fluid pressure, respectively } \\ S & : \text { Tensile force } \\ V & : \text { Flow velocity } \\ \Delta v, V_{0} & : \text { Unsteady and steady flow velocity, respectively } \\ w & : \text { Film displacement } \\ \mu & : \text { Fluid viscosity } \\ v_{f} & : \text { Kinematic viscosity } \quad\left(=\mu / \rho_{f}\right) \\ \rho & : \text { Film density } \\ \rho_{f} & : \text { Fluid density }\end{array}$

\section{Theoretical Stability Analysis}

\subsection{Modeling and Coordinate System}

Figure 1 and figure 2 show a traveling wave mode of the wave and an analytical model of the thin film subjected to the fluid flow together with the stationary coordinate system xyz and geometrical parameters considered in this model. The thin film is set in the middle of the narrow gap filled with fluid and is subjected to the fluid flow in y-direction. Tensile force is applied to the thin film. The equilibrium gap width in steady state is a constant, $H_{0}$, and thickness of the thin film is $h$. The uniform tensile force per unit width $S$ is applied in $\mathrm{x}$-direction. The fluid flow and tensile force are crossed at a right angle. 
In order to simplify the analytical model while not losing important factors relevant to the instability phenomena, the following assumptions are made with respect to the motion of the thin film and fluid flow. (i) The thickness of the thin film $h$ is small, and the thin film model is based on the Kirchhoff-Love's theory. (ii) The equilibrium position of the thin film is in the middle of the narrow gap. (iii) The gap width $H_{0}$ between the thin film and side wall is small compared with a length of the passage. (iv) The thin film displacement $w$ vibrating around the equilibrium position is smaller than the gap width $H_{0}$. (v) The fluid flow around the thin film is in the y-direction, and the fluid pressure gradient over the gap width is assumed to be zero, because the gap width is small. (vi) The fluid in the gap is incompressible and viscous, and separation of the fluid does not occur.

\subsection{Basic Equation of Structural Motion}

The equation of motion of the thin film subjected to the fluid flow is derived in terms of the transverse displacement $w$ and with respect to the stationary coordinate system xyz as follows:

$$
\begin{aligned}
\rho h \frac{\partial^{2} w}{\partial t^{2}}+C_{s} \frac{\partial w}{\partial t}+ & -S \frac{\partial^{2} w}{\partial x^{2}} \\
& +\frac{h^{3}}{12}\left(E+E^{*} \frac{\partial}{\partial t}\right)\left(\frac{\partial^{4} w}{\partial x^{4}}+2 \frac{\partial w^{4}}{\partial x^{2} \partial y^{2}}+\frac{\partial^{4} w}{\partial y^{4}}\right)=f,
\end{aligned}
$$

where $E$ is Young's modulus, $C_{\mathrm{s}}$ and $E^{*}$ are the external and internal structural damping coefficients, respectively. $f$ is fluid force acting on the thin film surface per unit area. $\rho$ and $h$ are the density and thickness of the thin film, respectively.

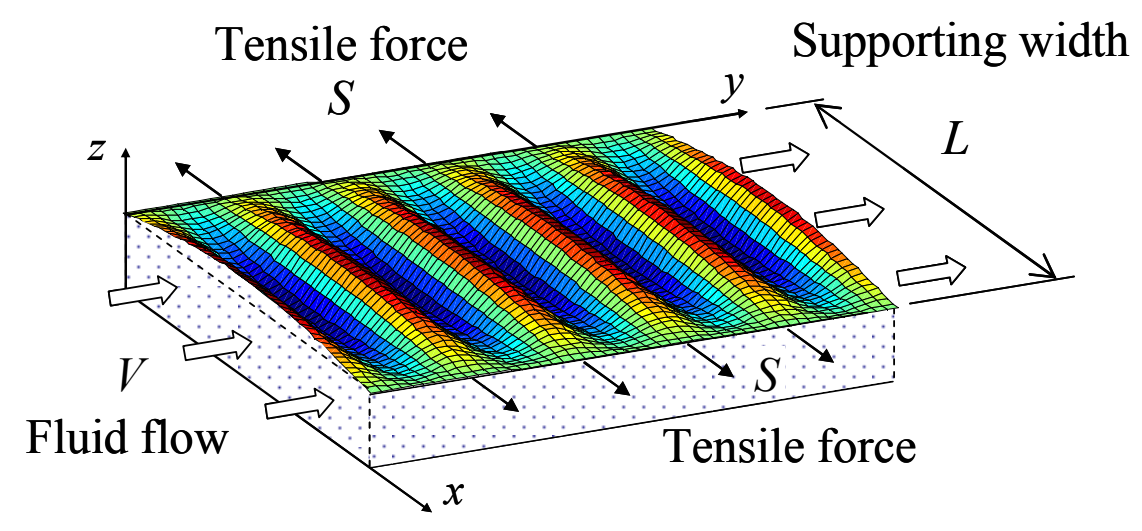

Figure.1 Traveling wave mode

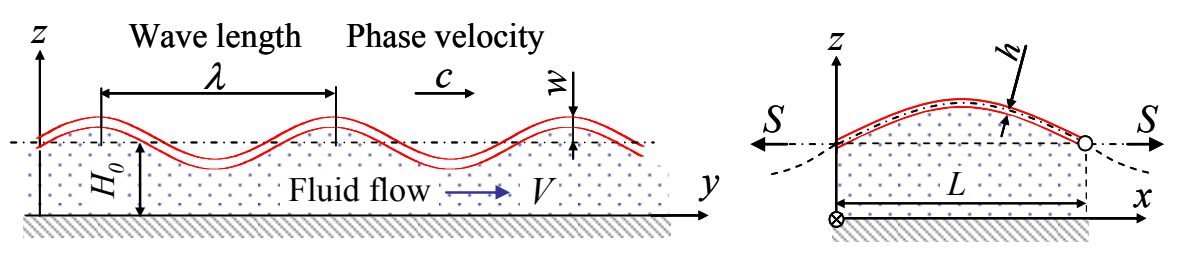

Figure.2 Analytical model 


\subsection{Basic Equations of Fluid Flow}

The equations of motion of the fluid flow around the thin film are derived by integrating the continuity and momentum equations of fluid motion over the gap width $H_{0}$ as follows:

$$
\begin{aligned}
& \frac{\partial H}{\partial t}+\frac{\partial}{\partial y}(H V)=0 \\
& \frac{\partial}{\partial t}(H V)+\frac{\partial}{\partial y}\left(H V^{2}\right)+\frac{H}{\rho_{f}} \frac{\partial P}{\partial y}+\frac{12 \mu}{\rho_{f}}\left(V-V_{0}\right)-\frac{1}{2}\left(f_{s}-f_{w}\right) V^{2}=0
\end{aligned}
$$

where $V$ is mean flow velocity over the gap width between the thin film and side wall. $V_{0}$ is steady flow velocity of the fluid flow in the narrow passage. $\mu$ is fluid viscosity. $f_{s}$ and $f_{w}$ are fluid friction coefficients on the surface of the thin film and side wall, respectively, and are assumed to be obtained as follows ${ }^{(20)}$ :

$$
f_{s}=f_{w}=\frac{1}{4}\left\{1.14-2 \log _{10}\left(\frac{\varepsilon_{H}}{D_{H}}+\frac{21.25}{\operatorname{Re}^{0.9}}\right)\right\}^{-2},
$$

where the friction coefficients $f_{s}$ and $f_{w}$ depend on the Reynolds number Re and the roughness of surface $\varepsilon_{H}$. The Reynolds number is defined as $\operatorname{Re}=V D_{H} / v_{f}$, where $D_{H}=2 H_{0}, \mu_{f}$ is kinematic viscosity of the fluid and $\varepsilon_{H}$ is roughness of the side wall respectively.

\subsection{Wave Equation}

In this section, a linear wave equation of bending wave motion of the thin film coupled with the fluid flow in the narrow gap is derived from the basic equations of motion of the thin film and fluid flow.

The gap width $H$ between the thin film and side wall is obtained using the steady equilibrium gap width $H_{0}$ and transverse displacement $w$ of the thin film as follows:

$$
H(x, y, t)=H_{0}+w(x, y, t) .
$$

Similarly, the flow velocity and fluid pressure are obtained by summation of the steady and unsteady terms as follows:

$$
\begin{aligned}
& V(y, t)=V_{0}+\Delta v(y, t), \\
& P(x, y, t)=P_{0}(y)+p(x, y, t),
\end{aligned}
$$

where $V_{0}$ and $\Delta v$ are steady and unsteady flow velocities, and $P_{0}$ and $p$ are steady and unsteady fluid pressures, respectively. Here the unsteady fluid pressure is induced by the motion of the thin film.

Substituting Eqs. (5), (6) and (7) into the basic Eqs. (2) and (3), the basic equations of the fluid flow are linearized with respect to the unsteady terms as follows:

$$
\begin{aligned}
& \frac{\partial \hat{w}}{\partial T}+\frac{\partial \Delta \hat{v}}{\partial Y}+\hat{V} \frac{\partial \hat{w}}{\partial Y}=0, \\
& \frac{\partial \Delta \hat{v}}{\partial T}+\hat{V} \frac{\partial \hat{w}}{\partial T}+2 \hat{V} \frac{\partial \Delta \hat{v}}{\partial Y}+\hat{V}^{2} \frac{\partial \hat{w}}{\partial Y}+\frac{12 \hat{\mu} \hat{h}}{\hat{\rho}} \Delta \hat{v} \\
&+\left(f_{w}-f_{s}\right) \hat{V} \Delta \hat{v}+\frac{1}{2}\left(f_{s}-f_{w}\right) \hat{V}^{2} \hat{w}+\frac{\hat{h}}{\hat{\rho}} \frac{\partial \hat{p}}{\partial Y}=0,
\end{aligned}
$$

where each term is expressed by dimensionless parameters by defining the following quantities: 


$$
\begin{aligned}
& T_{0}=\sqrt{\frac{\rho h H_{0}^{4}}{D}}, U_{0}=\frac{H_{0}}{T_{0}}, T=\frac{t}{T_{0}}, \hat{V}=\frac{V_{0}}{U_{0}}, \hat{L}=\frac{L}{H_{0}}, \hat{w}=\frac{w}{H_{0}}, \\
& X=\frac{x}{H_{0}}, Y=\frac{y}{H_{0}}, \hat{h}=\frac{h}{H_{0}}, \Delta \hat{v}=\frac{\Delta v}{U_{0}}, \hat{\rho}=\frac{\rho_{f}}{\rho}, \hat{S}=\frac{S H_{0}^{2}}{D}, \hat{p}=\frac{H_{0}^{3}}{D} p, \\
& \hat{\mu}=\mu \sqrt{\frac{H_{0}^{2}}{\rho h D}}, \hat{C}_{s}=C_{s} \sqrt{\frac{H_{0}^{4}}{\rho h D}}, \hat{f}=\frac{H_{0}^{3}}{D} f, \varepsilon=\frac{E^{*}}{E T_{0}}, D=\frac{E h^{3}}{12} .
\end{aligned}
$$

Similarly, the dimensionless basic equation of the thin film is obtained from Eq. (1) using the dimensionless parameters as follows:

$$
\frac{\partial^{2} \hat{w}}{\partial T^{2}}+\hat{C}_{s} \frac{\partial \hat{w}}{\partial T}-\hat{S} \frac{\partial^{2} \hat{w}}{\partial X^{2}}+\left(1+\varepsilon \frac{\partial}{\partial T}\right)\left(\frac{\partial^{4} \hat{w}}{\partial X^{4}}+2 \frac{\partial^{4} \hat{w}}{\partial X^{2} \partial Y^{2}}+\frac{\partial^{4} \hat{w}}{\partial Y^{4}}\right)=\hat{f}(X, Y, T),
$$

where $\hat{f}$ denotes the dimensionless fluid force obtained from the dimensionless unsteady fluid pressure $\hat{p}$ using following relationship:

$$
\hat{f}(X, Y, T)=\hat{p}(X, Y, T) .
$$

From these linearized equations, Eqs. (8), (9) and (11), a linear wave equation of bending wave motion of the thin film coupled with the fluid flow is obtained as follows:

$$
\begin{aligned}
& \left(\frac{\partial}{\partial T}+\hat{V} \frac{\partial}{\partial Y}\right)^{2} \hat{w}+\gamma\left(\frac{\partial}{\partial T}+\frac{\partial}{\partial Y}\right) \hat{w} \\
& =\frac{1}{\beta} \frac{\partial^{2}}{\partial Y^{2}}\left\{\frac{\partial^{2} \hat{w}}{\partial T^{2}}+\hat{C}_{s} \frac{\partial \hat{w}}{\partial T}-\hat{S} \frac{\partial^{2} \hat{w}}{\partial X^{2}}+\left(1+\varepsilon \frac{\partial}{\partial T}\right)\left(\frac{\partial^{4} \hat{w}}{\partial X^{4}}+2 \frac{\partial^{4} \hat{w}}{\partial X^{2} \partial Y^{2}}+\frac{\partial^{4} \hat{w}}{\partial Y^{4}}\right)\right\}
\end{aligned}
$$

where $\beta, \gamma$, and $\delta$ are defined by the following dimensionless parameters:

$$
\begin{aligned}
& \beta=\frac{\hat{\rho}}{\hat{h}}, \\
& \gamma=\frac{12 \hat{\mu} \hat{h}}{\hat{\rho}}+\left(f_{w}-f_{s}\right) \hat{V}, \\
& \delta=\hat{V}-\frac{\alpha}{\gamma},
\end{aligned}
$$

where $\alpha$ is defined by the following dimensionless parameters:

$$
\alpha=\frac{1}{2}\left(f_{s}-f_{w}\right) \hat{V}^{2} .
$$

From Eq. (13), it is found that the wave equation is composed two fluid-wave equations expressed by first and second-order differential equations and the structural wave equation derived from the equation of motion of the thin film. The first fluid-wave equation is derived from the inertia term of the basic equation of the thin film and the continuity equation of the fluid flow. The second fluid-wave equation is derived from the viscosity and friction loss terms of the basic equation of the thin film and the continuity equation of the fluid flow.

Moreover, in Eq. (13), $\gamma$ denotes a dimensionless parameter of the viscosity and friction loss of the fluid. $\beta$ denotes a dimensionless parameter which has the effect of the fluid-waves on the structural wave of the thin film. If $\beta$ is large, the fluid-waves are dominant compared with the structural wave traveling on the thin film. On the other hand, if $\beta$ is small, the structural wave is dominant compared with the fluid-waves. Thus, it is found that the dimensionless parameter $\beta$ is one of the most important parameter for the dynamic characteristics and stability of the wave. 


\subsection{Dispersion Relation and Stability Analysis}

In this section, the dispersion relation of the wave is derived from the wave equation, (13), to investigate the dynamic characteristics, phase velocity (traveling-wave velocity) and growth rate of the wave. The transverse displacement $w$ of the thin film is expressed as follows:

$$
\hat{w}=\hat{w}_{0} \sin \left(\frac{\pi X}{\hat{L}}\right) \exp \{i(\hat{\omega} T-k Y)\}
$$

Substituting Eq. (18) into the wave Eq. (13), the dispersion relation of the wave is obtained as follows:

$$
\begin{aligned}
\left(\beta+k^{2}\right) \hat{\omega}^{2}-2 k \beta \hat{V} \hat{\omega}-\left[\gamma \beta+\hat{C}_{S} k^{2}+k^{2} \varepsilon\left\{\left(\frac{\pi}{\hat{L}}\right)^{2}+k^{2}\right\}^{2}\right] i \hat{\omega} \\
+\left[\beta k^{2} \hat{V}^{2}-k^{2}\left\{\left(\frac{\pi}{\hat{L}}\right)^{2}+k^{2}\right\}^{2}-\hat{S} k^{2}\left(\frac{\pi}{\hat{L}}\right)^{2}\right]+i \gamma \beta \delta k=0 .
\end{aligned}
$$

The stability and dynamic characteristics of the wave generated on the thin film are dependent on the complex root, $\hat{\omega}$ obtained from Eq. (19), where $\operatorname{Re}[\hat{\omega}] / k$, real part of $\hat{\omega}$ divided wave number $k$, is denotes the phase velocity of the wave. If $\operatorname{Re}[\hat{\omega}] / k$ is positive, the wave is forward wave which propagates in the same direction as the fluid flow, and if $\operatorname{Re}[\hat{\omega}] / k$ is negative, the wave is backward wave which propagates in the opposite direction as the fluid flow. Moreover, $-\operatorname{Im}[\hat{\omega}]$, negative imaginary part of $\hat{\omega}$, denotes the growth rate of the wave. If $-\operatorname{Im}[\hat{\omega}]$ is positive, the wave grows exponentially in time, that is, an unstable wave occurs, and if $-\operatorname{Im}[\hat{\omega}]$ is negative, the wave is stable.

\section{Analytical Parameters}

Table 1 shows parameters used in the calculation for the stability analysis. These parameters correspond to the experimental parameters used in the other experimental study. In the calculations, the thin film is made of PET (polyethylene terephthalate), and the fluid in the gap between the thin film and side wall is air. Moreover, the external and internal structural damping coefficient are assumed $C_{\mathrm{s}}=10.0 \mathrm{Ns} / \mathrm{m}^{3}$ and $E^{*}=1.0 \times 10^{3} \mathrm{Ns} / \mathrm{m}^{2}$. In this conditions, dimensionless parameters are $\beta=2.46 \times 10^{-1}, \hat{C}_{s}=1.65 \times 10^{-2}, \varepsilon=9.01 \times$ $10^{-5}$ and $\hat{S}=2.40 \times 10^{3}$.

Table.1 Parameters used in the calculation

\begin{tabular}{|c|c|}
\hline Material of sheet & PET \\
\hline Fluid & Air \\
\hline Gap width $H_{0}$ & $5.0 \times 10^{-3}[\mathrm{~m}]$ \\
\hline Film width $L$ & $0.3,0.4,0.5,0.6,[\mathrm{~m}]$ \\
\hline Film thickness $h$ & $16.0 \times 10^{-6}[\mathrm{~m}]$ \\
\hline Tensile force $S$ & $50,100,150,200[\mathrm{~N} / \mathrm{m}]$ \\
\hline Film Young's modulus $E$ & $3.0 \times 10^{9}[\mathrm{~Pa}]$ \\
\hline Film density $\rho$ & $1.4 \times 10^{3}\left[\mathrm{~kg} / \mathrm{m}^{3}\right]$ \\
\hline Fluid density $\rho_{\mathrm{f}}$ & $1.1\left[\mathrm{~kg} / \mathrm{m}^{3}\right]$ \\
\hline Fluid viscosity $\mu$ & $18.2 \times 10^{-6}\left[\mathrm{Ns} / \mathrm{m}^{2}\right]$ \\
\hline
\end{tabular}




\section{Analytical Results}

In this section, first, the effect of the fluid flow on the phase velocity and growth rate of the wave generated on the thin film is presented. Next, the effect of the dissipation terms which include the fluid viscosity, wall friction and structural damping is presented.

\subsection{Phase Velocity and Growth Rate of Wave}

Figure 3 (a) and (b) show the phase velocity $\operatorname{Re}[\hat{\omega}] / k$ and growth rate $-\operatorname{Im}[\hat{\omega}]$ of the wave generated on the thin film subjected to the fluid flow in the narrow passage as a function of the flow velocity $\hat{V}$. In this figure, three types of curve denote the results of wave number $k=1.2,1.6,2.0$, respectively. From Fig.3 (a), it is seen that there are forward and backward waves for each wave number at the flow velocity of zero, and vary with increasing flow velocity. The phase velocity of the backward wave progressively goes to zero. After the phase velocity of the backward wave has become zero, the backward wave becomes forward wave, and its phase velocity increases as the flow velocity increases. From Fig.3 (b), it is seen that unstable waves occurs because growth rate of the forward wave becomes positive, and that the waves occur as a type of traveling wave.

Figure 4 (a) and (b) show the dispersion relation of waves generated on the thin film subjected to the fluid flow, where (a) and (b), respectively, show the phase velocity $\operatorname{Re}[\hat{\omega}] / k$ and growth rate $-\operatorname{Im}[\hat{\omega}]$ of wave as a function of the wave number $k$. In this figure, four types of curve denote the results at the flow velocity $\hat{V}=5,10,15,20$.

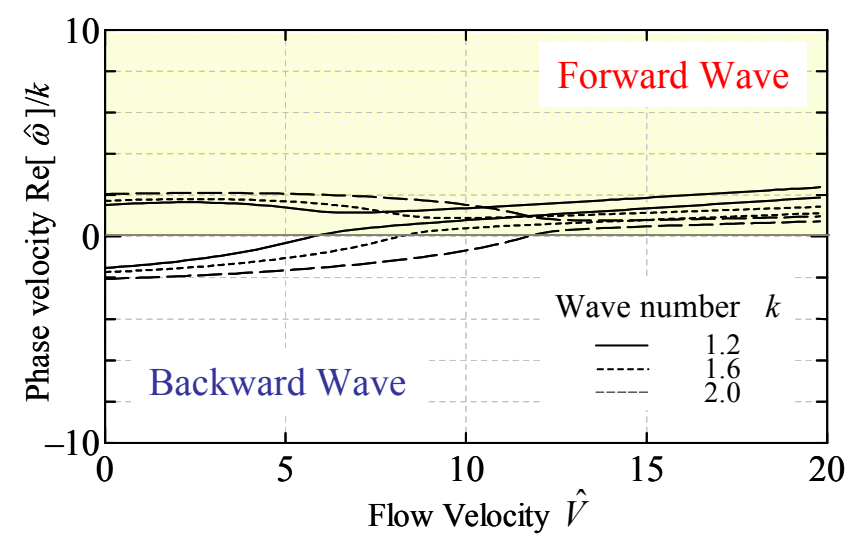

(a) Phase velocity $\operatorname{Re}[\hat{\omega}] / k$ with changing flow velocity $\hat{V}$

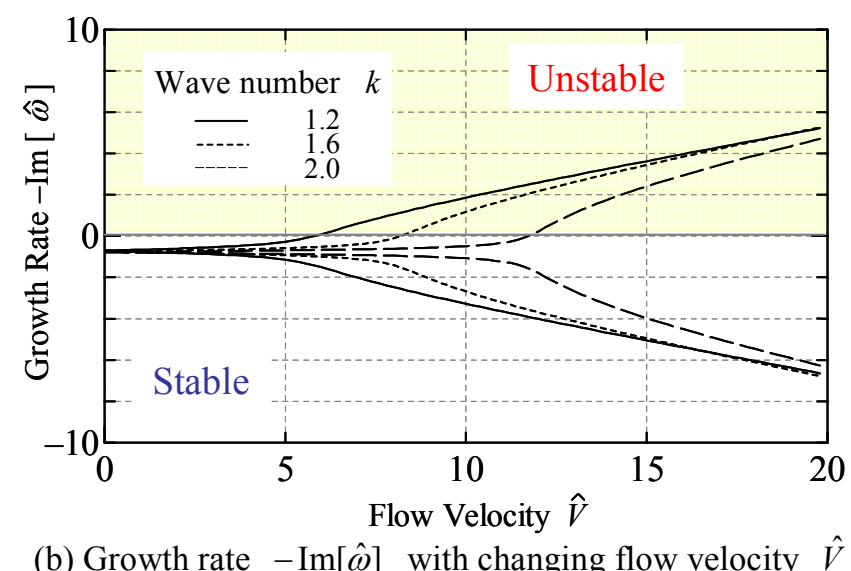

Figure.3 Variations of phase velocity $\operatorname{Re}[\hat{\omega}] / k$ and growth rate $-\operatorname{Im}[\hat{\omega}]$ with changing flow velocity $\hat{V}$ 
From Fig.4 (a), it is seen that the phase velocity and growth rate of the wave vary greatly with increasing flow velocity and the wave generated on the thin film is a dispersive wave because the flow velocity is greatly dependent on the wave number. Also, the most unstable wave number increase as the fluid flow increases, as shown in Fig.4 (b).

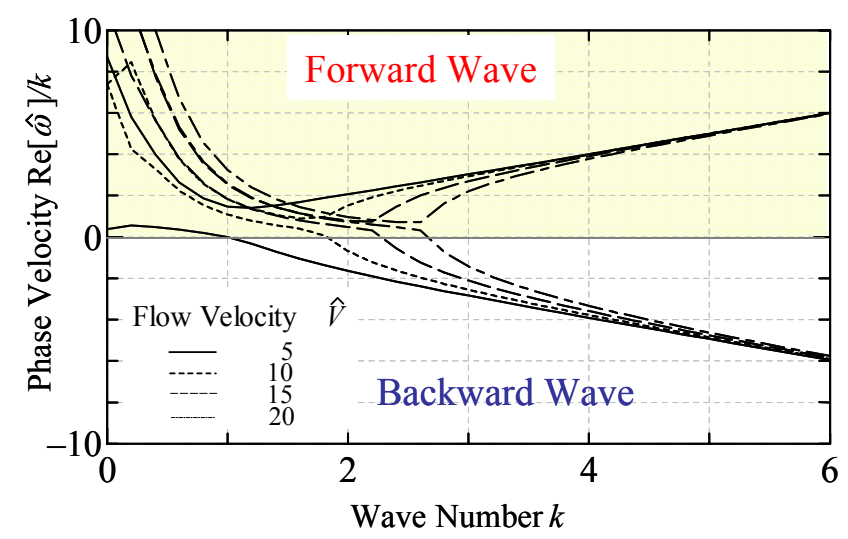

(a) Phase velocity $\operatorname{Re}[\hat{\omega}] / k$ with changing wave number $k$

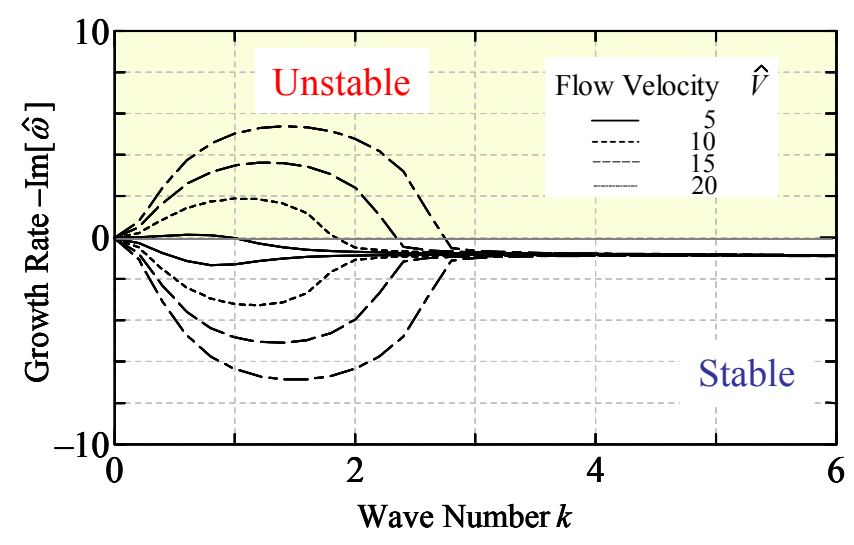

(b) Growth rate $-\operatorname{Im}[\hat{\omega}]$ with changing wave number $k$

Figure.4 Dispersion relation of wave

\subsection{Phase Velocity and Growth Rate of Wave Without Dissipation Effects}

Figure 5 (a) and (b) shows the phase velocity $\operatorname{Re}[\hat{\omega}] / k$ and growth rate $-\operatorname{Im}[\hat{\omega}]$ of the wave generated on the thin film as a function of the flow velocity $\hat{V}$ ignoring the dissipation terms which include the fluid viscosity, wall friction and structural damping ( $\left.\gamma=0, \hat{C}_{s}=0, \varepsilon=0\right)$. In this figure, three types of curve denote the results of wave number $k=1.2,1.6,2.0$, respectively.

From Fig.5 (a) and (b), the unstable wave occurs as a type of the forward wave with increasing the flow velocity. By comparison between Fig. 3 and Fig. 5 , it can be seen that the unstable wave occurs because of the right side of the Eq.(12) which describe the structural wave motion is coupled with the first term of Eq.(12) which describe the fluid-waves motion. Moreover, after the backward wave becomes a forward wave, the phase velocity of the forward waves increase linearly in the higher flow velocity region. 


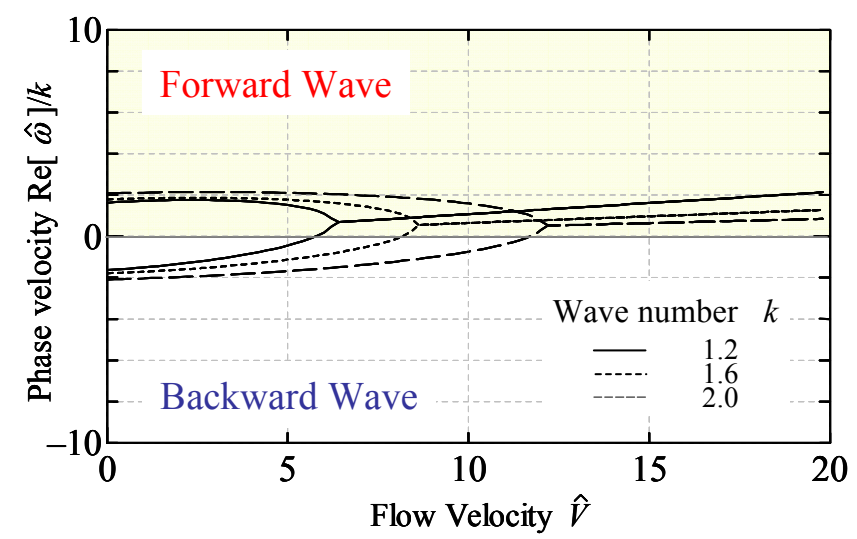

(a) Phase velocity $\operatorname{Re}[\hat{\omega}] / k$ with changing flow velocity $\hat{V}$

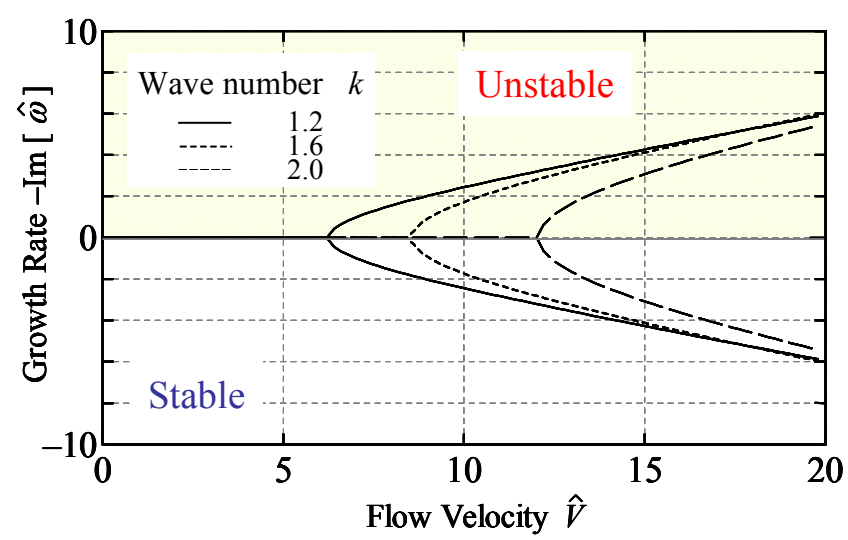

(b) Growth rate $-\operatorname{Im}[\hat{\omega}]$ with changing flow velocity $\hat{V}$

Figure.5 Variations of phase velocity $\operatorname{Re}[\hat{\omega}] / k$ and growth rate $-\operatorname{Im}[\hat{\omega}]$ with changing flow velocity $\hat{V}$ (without dissipation effects)

\section{Unstable Region}

\subsection{Effects of Tensile Force and Film Supporting Width}

Figure 6 shows the unstable region of the wave with changing the flow velocity as a function of the tensile force $\hat{S}$. In this figure, four types of curve denote the results of the tensile force $\hat{S}=1.2 \times 10^{3}, 2.4 \times 10^{3}, 3.6 \times 10^{3}, 4.8 \times 10^{3}$ respectively. It can be seen that the unstable region of wave number becomes narrower with increasing the tensile force, and the critical flow velocity increases with increasing the tensile force. The unstable region of the wave number becomes broader with increasing the fluid flow. Moreover, it can be seen that the most unstable wave number is about $k=0.5$.

Figure 7 shows the unstable region of the wave with changing the flow velocity as a function of the film supporting width. In this figure, four types of curve denote the results of the film supporting width $\hat{L}=60,80,100,120$ respectively. It can be seen that the unstable region of wave number becomes broader with increasing the film supporting width, and the critical flow velocity decreases with increasing the film supporting width. The unstable region of the wave number becomes broader with increasing the flow velocity. Moreover, it can be seen that the most unstable wave number is about $k=0.5$. 


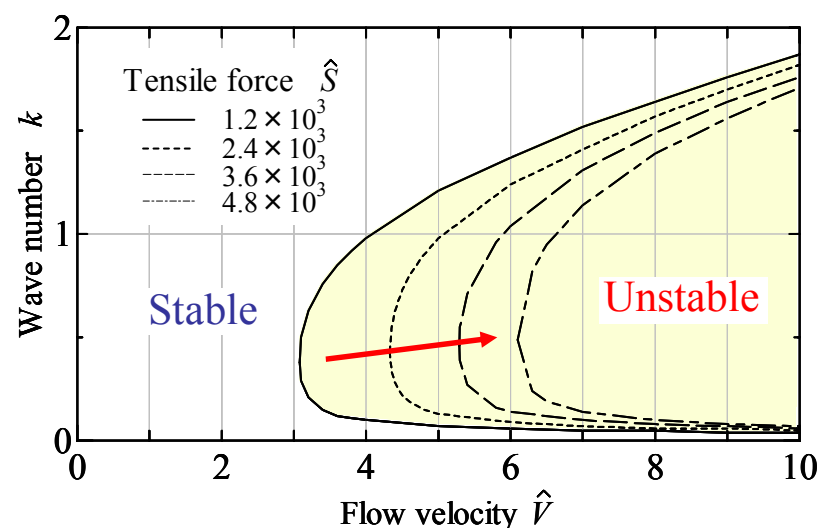

Figure.6 Variation of unstable region with changing tensile force $\hat{S}$

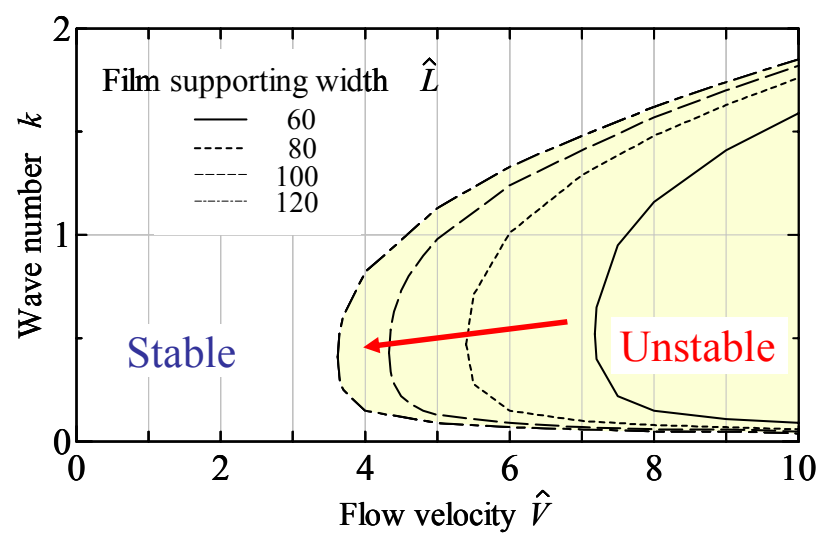

Figure.7 Variation of unstable region with changing film supporting width $\hat{L}$

\subsection{Effect of Structural Damping}

Figure 8 shows the unstable region of the wave with changing the flow velocity as a function of the external damping coefficient $\hat{C}_{s}$. Figure 9 shows the unstable region of the wave with changing the flow velocity as a function of the internal damping coefficient $\varepsilon$. In these figures, three types of curve denote the results of the internal damping coefficient $\hat{C}_{s}=1,10,100$ and external damping coefficient $\varepsilon=10^{2}, 10^{4}, 10^{6}$, respectively. And the symbol is from experiments. From these figures, the unstable region of wave number becomes broader with increasing structural damping, and the critical flow velocity decreases with increasing the structural damping. But the effect of the structural damping on the most unstable wave number and critical flow velocity does not change greatly. That is, the effect of the structural damping is relatively insensitive to the most unstable wave number and critical flow velocity of the unstable wave.

In the experiments, we observed the unstable wave whose wavelength is $\lambda=0.01[\mathrm{~m}]$, and the critical flow velocity is $V=6.5[\mathrm{~m} / \mathrm{s}]$. The wave number and non-dimensional critical flow velocity are obtained as follows:

$$
\begin{aligned}
& k=\frac{1}{\hat{\lambda}}=\frac{H_{0}}{\lambda}=0.5, \\
& \hat{V}_{c r}=\frac{V_{c r}}{V_{0}}=4.5 .
\end{aligned}
$$

Comparing the theoretical and experimental results, theoretical results of the most unstable wave number and critical flow velocity are in good agreement with the experimental results, hence the effect of the structural damping is relatively insensitive to the most unstable wave number and critical flow velocity of the unstable wave. 


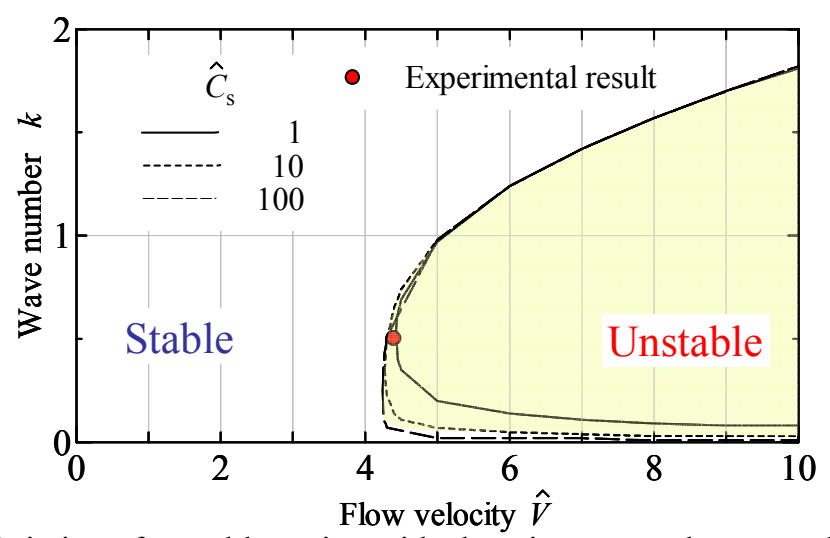

Figure.8 Variation of unstable region with changing external structural damping $\hat{C}_{s}$

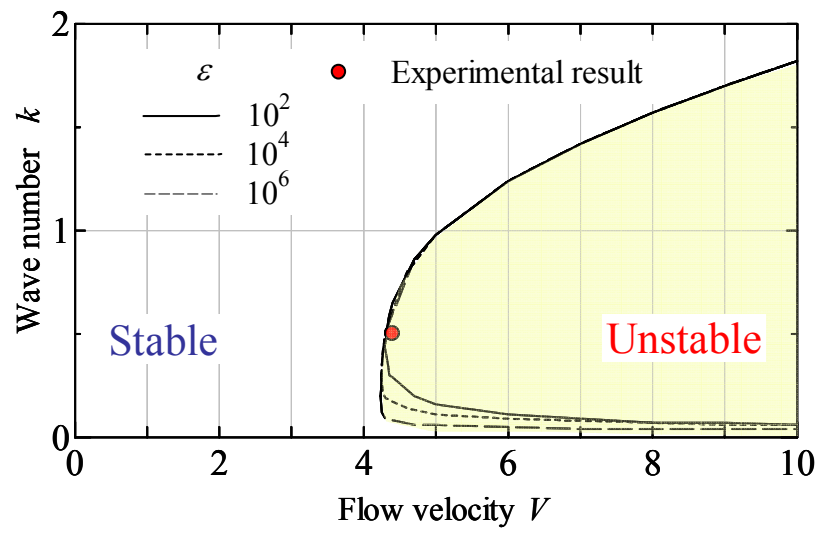

Figure.9 Variation of unstable region with changing internal structural damping $\varepsilon$

\section{Conclusions}

The theoretical stability analysis of unstable wave generated on the thin film subjected to the fluid flow in the narrow gap was developed. In the stability analysis, we examined the effect of the fluid flow, tensile force, film supporting width and structural damping on the stability and dynamic characteristics of the wave. The following main conclusions were obtained:

(1) The analytical results clarified the dispersion relation of the wave motion generated on the thin film subjected to the fluid flow in the narrow gap.

(2) The traveling wave mode flutter occurs to the thin film due to the fluid flow in the narrow gap when the flow velocity becomes higher.

(3) The unstable region of wave number becomes narrower with increasing the tensile force, and becomes broader with increasing the flow velocity. The critical flow velocity increases with increasing the tensile force. On the other hands, the unstable region of wave number becomes broader with increasing the film supporting width, and becomes narrower with increasing the flow velocity. The critical flow velocity decreases with increasing the film supporting width.

(4) The most unstable wave number is about $k=0.5$. The most unstable wave number and critical flow velocity are in good agreement with the experimental results.

\section{References}

(1) F. Inada, S. Hayama, "A Study on Leakage-Flow-Induced Vibrations $\left(1^{\text {st }}\right.$ Report, Fluid-Dynamic Forces Acting on the Walls of a One-Dimensional, Narrow, Tapered Passage)": JSME Int. J. Series III, 31, pp.39-47, 1998.

(2) F. Inada, S. Hayama, "A Study on Leakage-Flow-Induced Vibrations. Part 1: 
Fluid-Dynamic Forces and Moments Acting on the Walls of a Narrow Tapered Passage": Journal of Fluids and Structures, 4, pp.395-412, 1990.

(3) F. Inada, S. Hayama, "A Study on Leakage-Flow-Induced Vibrations. Part 2: Stability Analysis and Experiments for Two-Degree-of-Freedom Systems Combining Translational and Rotational Motions": Journal of Fluids and Structures, 4, pp.413-428, 1990.

(4) H. Nagakura, S. Kaneko, "The Stability of a Cantilever Beam Subjected to One-Dimensional Leakage Flow (in Japanese)": Transactions of the JSME. Series C, 58-546, pp.352-259, 1992.

(5) N. Yamaguchi, K Yokota, Y. Tsujimoto, "Flutter Limits and Behavior of a Flexible Thin Sheet in High-Speed Flow ( $1{ }^{\text {st }}$ Report, Analytical Method for Prediction of the Behavior)": Journal of Fluids Engineering, 122, pp.65-73, 2000.

(6) N. Yamaguchi, T. Sekiguchi, K. Yokota, Y. Tsujimoto, "Flutter Limits and Behavior of a Flexible Thin Sheet in High-Speed Flow ( $2^{\text {nd }}$ Report, Experimental Results and Predicted Behaviors for Low Mass Ratios)": Journal of Fluids Engineering, 122, pp.74-83, 2000.

(7) Y. Watanabe, S. Suzuki, M. Sugihara, Y. Sueoka, "An Experimental Study of Paper Flutter": Journal of Fluids and Structures, 16, pp.529-542, 2002.

(8) Y. Watanabe, K. Isogo, S. Suzuki, M. Sugihara, “A Theoretical Study of Paper Flutter": Journal of Fluids and Structures, 16, pp.543-560, 2002.

(9) C. Shin, J. Chung, W. Kim, "Dynamic Characteristics of the Out-of-Plane Vibration for an Axially Moving Membrane”: Journal of Sound and Vibration, 286, pp.1019-1031, 2005.

(10) X. Wu, S. Kaneko, "Linear and Nonlinear Analysis of Sheet Flutter Induced by Leakage Flow: Journal of Fluids and Structures, 20, pp.927-948, 2005.

(11) L. Huang, "Flutter of Cantilevered Plates in Axial Flow": Journal of Fluids and Structures, 9, pp.127-147, 1995.

(12) F. Inada, "A Study on Leakage-Flow-Induced Vibrations of a Flexible Structure (A Stability Analysis of One Dimensional Flexible Plate and a Consideration Self-Excited Oscillation Mechanisms) (in Japanese)": Transaction of the JSME. Series C, 66-645, pp.1475-1482, 2000.

(13) C. A. Evrensel, A. Kalnins, "Response of Compliant Slab to Inviscid Incompressible Fluid Flow": Journal of Acoustical Society of America, 78, pp.2034-2041, 1985.

(14) R. J. Hansen, D. J. Huston, C. C. Ni, “An Experimental Study of Flow-Induced Waves on a Flexible Surface”: Journal of Sound and Vibration, 68, pp.317-334, 1980.

(15) P. W. Carpenter, A.D.Garrad, "The Hydrodynamic Stability of Flow Over Kramer-Type Compliant Surface. Part 1. Tollmien-Schlichting Instabilities": Journal of Fluid Mechanics, 155, pp.465-510, 1985.

(16) P. W. Carpenter, A. D. Garrad, "The Hydrodynamic Stability of Flow Over Kramer-Type Compliant Surface. Part 2. Flow-Induced Surface Instabilities": Journal of Fluid Mechanics, 170, pp.199-232, 1986.

(17) Y. B .Chang, P. M. Moretti, "Flow-Induced Vibration of Free Edges of Thin Film": Journal of Fluids and Structures, 16, pp.989-1008, 2002.

(18) M. Watanabe, N. Kobayashi, "Stability Analysis of Flow-Induced Waves of a Flexible Sheet Moving in a Fluid-Filled Narrow Space with Shear Fluid Flow ( $1^{\text {st }}$ Report, In a Case of Moving Infinity Long Sheet) (in Japanese)": Transaction of the JSME. Series C, 67-658, pp.1771-1778, 2001.

(19) M. Watanabe, N. Kobayashi, "Dynamics and Stability Analysis of Axially Moving Web Subjected to Shear Fluid Flow in Narrow Space (in a Case of Finitely Long Space) (in Japanese)": Transaction of the JSME. Series C, 67-664, pp.3722-3729, 2001.

(20) F. Axisa, J. Antunes, "Flexural Vibrations of Rotors Immersed in Dense Fluids Part I, Theory”: Journal of Fluids and Structures, 6, pp.3-21, 1992 